\title{
Application of TRIZ Theory in Patternless Casting Manufacturing Technique
}

\author{
Weidong Yang, Dequan Gan, Ping Jiang, and Yumei Tian \\ School of Mechanical Engineering, Hebei University of Technology, \\ 300130, Tianjin, China \\ \{Weidong.Yang, DeQuan.Gan, Ping.Jiang, Yumei.Tian, \\ ywd_stephen\}@163.com
}

\begin{abstract}
The ultimate goal of Patternless Casting Manufacturing (referred to as PCM) is how to obtain the casts by casting the sand mold directly. In the previous PCM, the resin content of sand mold is much higher than that required by traditional resin sand, so the casts obtained are difficult to be sound and qualified products, which limits the application of this technique greatly. In this paper, the TRIZ algorithm is introduced to the innovation process in PCM systematically.
\end{abstract}

Keywords: TRIZ, Patternless Casting Manufacturing, Sand Mold, Resin.

\section{Introduction}

Patternless Casting Manufacturing technique (referred to as PCM) is the integrated system of multi-disciplinary knowledge and technology such as computer technology, NC technology, mechanical technology, electronics technology, materials technology and so on. This technology has the very strong industrial application background which is the product of market competition and technology development.

PCM technique is based on the discreteness and deposition principle of rapid prototyping, but it is completely different from the traditional casting mold manufacturing method [1]. In short the basic principle of the previous PCM process is illustrated as Fig.1. The procedure is that firstly, casting mold CAD model is obtained from part CAD model. The profile data of layer cross section come from casting mold CAD data, and then the layer information generates control information. The second nozzle sprays the catalyst along the same path, after the first nozzle sprays the binder accurately under the control of a computer. The binder solidifies the sand layer by layer until finishing building the model. The common place of the binder and catalyst interaction is solidified together; others without the binder and catalyst are still loose sand. The next layer is bonded after the layer is coalesced. The spatial entity is formed until all of layers are bonded. The sand without binder is still dry sand easy to clean out. A casting model that has a defined shape and wall-thickness is obtained after cleaning away the dry sand. The dope is coated or imbedded on the inner surface of sand model, which is used to cast metal parts. 

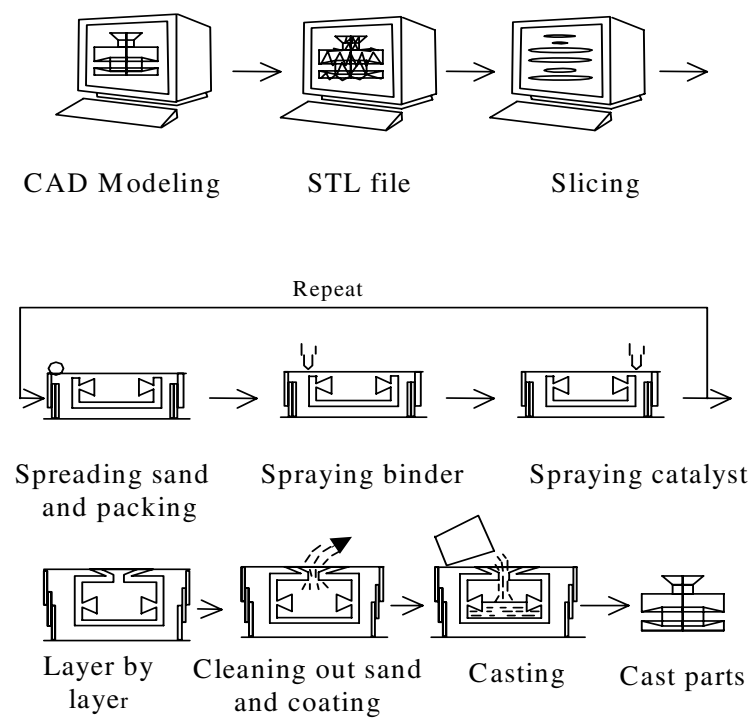

Fig. 1. Schematic of principle of PCM technique

In the previous PCM, the resin content of sand mold is much higher than that required by traditional resin sand, so the casts obtained are difficult to be sound and qualified products [2]. Therefore, a theory is needed to how to solve the high resin content in PCM.

TRIZ (Theory of Inventive Problem Solving) thought that the evolution of engineering system is not a stochastic process, but it is controlled by certain objective laws [3-4]. TRIZ pointed out that project conflict is the reason for creative design, and proposed that the evolution rule of engineering system show the evolution direction of engineering system. Therefore, the persons maintain the correct thinking in the creative invention and the design to avoid the blind searching.

\section{Creative Design Algorithm}

Creative design algorithm is the important analytical tool in TRIZ theory. It provides the general principles of solving engineering conflict creatively. Its steps are as followed [5-6]:

- Problem refining

- This step causes the question clarification, which makes a vague and unclear question to be descripted to be an explicit question. The refining process requires the system should maintain unchanged as far as possible, but can achieve the required functions.

- Description of engineering conflict

- The project conflict of the question should be discovered. The important parameters needed changing and other parameters affected are determined clearly. 
- Solving model of problem

- A simple idea to solve the problem is given.

- Indicating conflict domain

- The domain of engineering conflict is specified, which causes the question to be more explicit. The resources and the material used for solving the engineering conflict should also be considered and evaluated.

- Description of ideal result

- An Ideal result is expected. In order to realize this ideal result, key components of the system in the conflict domain should have physical characteristics of the conflict which is called the physical conflict.

- Method to overcome physical conflict

- There are three methods to overcome the physical conflict. The first method is to separates the conflict characteristic from the time and the space. The second method is to transform system. The last one is to use the phase-change of the material or the physical-chemistry transformation the material to solve the conflict.

To resolve the conflict, we must apply the above three methods flexibly, take full advantage of existing resources, and have a wealth of physical, chemical and geometric knowledge, etc.. In the TRIZ theory, this knowledge is the physical, chemical and geometric effects database.

\section{Application of TRIZ in PCM}

\subsection{Proposed Problem}

In the previous PCM, the resin and curing agent were sprayed in the surface of sand according to the planning path separately by continuous nozzles. The method of spraying resin to achieve the sand particles bonding together makes the resin content of sand mold with a high level, thus resulting the casts have the defect and affecting the casting process proceeding smoothly. Therefore, how to reduce the resin content is the one of important goals.

Using the jetting method to spray the resin causes the resin content high. Although the methods to reduce the resin content from filling and post-processing in the previous PCM was proposed, but still could not reduce the resin content to meet the demand required by casting [2].

The problem is clarified: how to reduce the resin content in the casts fundamentally is the PCM true realization.

\subsection{Description of Engineering Conflict}

The cast manufacture is the process of forming. Forming is to use the certain methods of energy to organize materials orderly to be three-dimensional entity (parts) with geometric shape and a certain functions. From the flowchart of traditional sand mold may see that has had the pattern, which has determined the geometrical shape of the casts. The process of manufacturing the patterns, assembling the patterns, and putting the patterns in the sand box in essence has the geometrical information of the casts. The sand is mixed by the resin and curing agent by a certain percentage evenly, and 
poured into the sand box with the patterns and pressed .After the mold sand solidifying completely, the patterns are taken out, then the sand mold for casting is obtained. The process of forming includes mixing the sand, pouring the sand, solidifying the sand mold.

The mixer is used to achieve the resin and the curing agent to package the sand surface by rolling in the traditional resin sand; while in the previous PCM the resin and the curing agent are sprayed selectively, the package of the sand surface is achieved by establishing the liquid bridge between the sand particles. In this stage, the combination of RP principle with the traditional resin sand technique. However, due to the high resin content of casting mold, the casts have high rejection rate. It is difficult to use the PCM in the real production.

In summary, the engineering conflict is: the traditional casting manufacturing should make the patterns. The cycle of the pattern manufacturing accounts for the whole process cycle too large which causes its flexibility too low. Mixer can make the resin content of the cast to meet the requirements of casting, but the resin sand by mixing the resin, the curing agent and the sand in the mixer cannot be used for the RP processes. The previous PCM realized the RP principle, but the resin content is high because of using the jetting method to spray the resin.

\subsection{Solution of the Problem}

The purpose of forming sand mold is to find a way not to need manufacturing the patterns and to satisfy the requirements of resin content by traditional casting.

\subsection{Conflict Domain and Resource Analysis}

The original sand, binder and curing agent are still effective resources in forming process which should be applied in new technique. Only the application of these traditional resources makes the new technique closer to the current industrial production, speed up and facilitate the commercialization process. The contradiction between using the jetting method to spray resin and the requirement of the resin content in traditional cast is conflict domain of this problem.

\subsection{Ideal Result}

In order to ensure the purpose of forming, the resin sand is got by mixing the original sand and binder in mixer used by traditional resin sand. This will ensure the requirement of the resin content in the sand mold. The curing agent is controlled precisely according the geometry information of casting mold. Other factors affecting casting may maintain as far as possible with the traditional forming consistent. Thus the casting mold made by the new technique can be cast directly.

\subsection{Conflict and Overcome}

In the previous PCM the merits of traditional resin are abandoned completely, only to consider using selectively scanning method to spraying resin. To fundamentally reduce the resin content of casting mold, the resin jetting method used in the RP 
technology to achieve the connection between sand particles is separated from the previous PCM technology, which is the most critical and the first step in the new PCM technique. The resin and sand mixing process in jetting is separated through the method of time and space separation, the problem of the high resin content in previous PCM should not be limited to solve by the use of RP technology. The mixing process in traditional resin sand is applied to the new PCM. The original sand and resin are mixed in the mixer to be the resin sand in accordance with the requirements of a certain proportion [7].

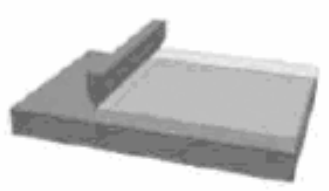

(1) Laying resin-bonded sand

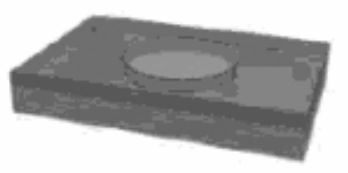

(4) Repeating step 1-3

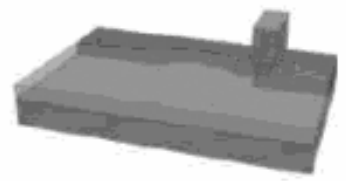

(2) Spraying catalyst

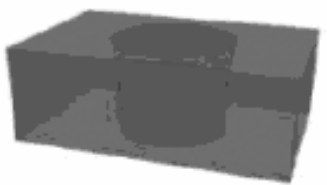

(5) Finishing the forming

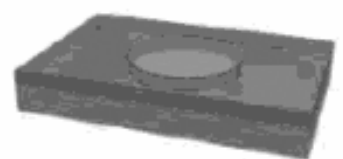

(3) Heating the sand surface

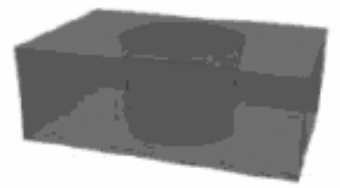

(6) Post-processing

Fig. 2. Schematic of new PCM process

Similar with the traditional self-hardening sand, in the new PCM process the resin and the sand is mixed to obtain the resin-bonded sand according to their proportion requirements in the high-speed blender. The resin-bonded sand which does not contain the catalyst is different from the traditional resin-bonded self-hardening sand. Combing the characteristics of RP technology, the catalyst is sprayed in the casting region selectively by the jetting device according to the layer information, which is reacted to the resin-bonded sand. Thus the sand containing the catalyst and the resin is solidified. In order to improve the efficiency of building, the heating device is used to heat the entire surface of resin-bonded sand. One layer upon layer is carried on; the casting mold is obtained according to the requirements (as shown in Fig.2).

The resin content of casting mold reduces fundamentally by applying the process of mixing of the resin and the original sand in PCM flexibility.

\section{Conclusion}

In PCM process the key is how to use the creative problem-solving methods to solve the problem. The practice shows that the innovative theory and algorithm are used in PCM which solve the limitation of the previous PCM. A new PCM process can be achieved. 


\section{Acknowledgments}

This research is supported in part by the Natural Science Foundation of Tianjin under Grant Numbers 07JCZDJC08900, the Key Project of the Ministry of Science and Technology of the People's Republic of China under Grant Numbers 2008IM030100, and the science and technology key project of Hebei Province under Grant Numbers $09212102 \mathrm{D}$.

\section{References}

1. Du, Z.: Study and Development of Casting Mold Manufacturing Directly Driven by CAD Model (PhD dissertation). Tsinghua University, Beijing (1997) (in Chinese)

2. $\mathrm{Xu}, \mathrm{D}$.: Study and Development of Patternless Casting Manufacturing and Machine $(\mathrm{PhD}$ dissertation). Tsinghua University, Beijing (2002) (in Chinese)

3. Altahuller, G.: The Innovation Algorithm, TRIZ, Systematic Innovation and Technical Creativity. Worcester Technical Innovation Center, INC (1999)

4. Tan, R.: Innovative design-TRIZ: Theory of Inventive Problem Solving. Mechanical Industry Press, Beijing (2002) (in Chinese)

5. Tan, R.: Process of Some Problems in Product Design for Innovation. Chinese Journal of Mechanical Engineering 39(9), 11-16 (2003) (in Chinese)

6. Tan, R., Zhang, Q., Ji, C.: The Law and Routes of System Evolution in TRIZ and the Application. Industrial Engineering and Management 8(1), 34-36 (2003) (in Chinese)

7. Yang, W.: Research on Patternless Casting Manufacturing Based on Sand Multiple-Package (PhD dissertation). Tsinghua University, Beijing (2003) (in Chinese) 